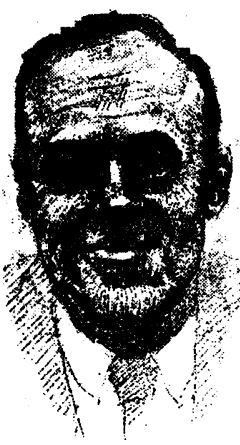

$\mathrm{T}$ he heart is grossly enlarged," said the postgraduate student after completing his examination, while a multinational force of his colleagues crowded around the patient's bed.

"Would you like to have an enlarged

heart?" the instructor snapped.

"No, sir," answered the student, taken aback by the unexpected vehemence.

"Then don't say it in front of the patient," said the instructor.

The year was 1960. The instructor was Paul Wood, at that time doyen of British cardiology. It was also the time when doctors still deemed it imprudent to frighten their patients with bad news. Syphilis was still the specific disease, a cancer was a lump, patients were not told their blood pressure readings, and physicians spoke of "accelerated" hypertension so their patients would not think they had a malignancy.

Since that time the bedside manners have changed. Doctors no longer speak Latin to conceal the diagnosis. They tell what the blood pressure is, draw little sketches of malignant tumours, and let patients read their charts. For informed consent for a barium enema they now must list every possible danger lurking along the way to the $x$ ray department: "You may fall down the elevator shaft; you may get stuck between floors and develop hypernatraemic dehydration; you may trip on a banana skin because the cleaners are out on strike; you may die of passive smoke inhalation; they may mistakenly give you calomel instead of barium; the stuff may go up or down the wrong way; they may perforate your bowel; the barium may get absorbed and you may develop hypokalaemia; you may never make it back to your bed; or the computer may reassign your bed and you may spend the night lying on the floor or sharing a bed with a stranger."

The medical literature is also replete with pronouncements by ethicists, lawyers, wise physicians, and people with cancer on how to impart bad news and offer truth without devastating the patient. These articles, often interspersed with passages from The Death of Ivan Ilych, emphasise that paternalism is dead and that patients need to know the truth so they can make appropriate arrangements. The doctor is variously advised to be detached but compassionate, not impersonal, not afraid

\section{Telling the patient}

to show emotion. Privacy is desirable, good eye contact recommended. The doctor should preferably be seated and should consider taking off his or her white coatespecially if it is blood stained. Doctors are advise to speak slowly and deliberately, choosing their words carefully. "X has died," for example, is thought to sound more humane that " $\mathrm{X}$ is dead."

Other articles point out that the patient has a right but not a duty to hear the bad news. Accordingly the doctor must probe gently, in what has been characterised as a series of complicated dance steps between doctor and patient, to determine how much the patient does want to know. Cultural differences are worth bearing in mind, doctors from eastern and southern Europe being more evasive and patients less inclined to want to hear the truth.

Such was the case of an elderly man from Cyprus-about to go home after a laparotomy -when his doctor brusquely told him that he had cancer, that he hoped it had been all removed, that it could recur, that he had a good chance of living many years, but that again he may not. The patient was incensed. He said that he had not asked and did not want to know. He promptly changed doctors and lived many years after this encounter. GEORGE DUNEA, attending physician, Cook County Hospital, Chicago, USA

\title{
Privileged men?
}

\section{L} ast year (BMF;309:350) Minerva expressed satisfaction that the causes of the relative longevity of Greek men compared with other Europeans had been elucidated. The traditional Mediterranean diet may indeed be part of the answer, but I believe there are other important factors, some of which are now changing, which have favoured Greek men from cradle to grave.

In the past the birth of a Greek male child was an occasion for rejoicing, congratulations, and celebrations whereas the birth of a daughter was usually met with the consolation, "Never mind! May she be well and the next one a son." The boy is brought up to believe that he is the centre of the universe, with his mother, grandmother, and sisters revolving around him and ministering to his every need. During his school years, shyness being unknown, he develops a wide circle of friends which coalesces in his teens to his own personal "parea"-inadequately translated as peer group or gang-a source of mutual unquestioning loyalty and affection that is his rock of support during the storms of adolescence.

If he can survive the hazards of youth that nowadays take the form of road accidents, drugs, and a rising incidence of AIDS, he will make a relatively late marriage to a much younger wife, a union that is considerably more likely to endure than those in other European countries. Although divorce is increasing, he can gain legitimate respite from the everyday irritations of marriage by escaping to the local cafeneon where his ruffled feelings will be soothed by his all male parea.

Throughout his life he is expected and encouraged to express strong emotions by singing, dancing, shouting, laughing, and weeping and through constant physical contact; hugging, kissing, holding hands, and caressing are normal components of everyday human contact for both men and women, as is constant, eloquent, verbal communication. Being alone is equated with loneliness and is considered the worst of human afflictions, whereas good company, together with food and wine, is the elixir of life.

He often holds down more than one job, which he needs in order to fulfil his extensive family obligations, and is also more likely than other European men to be employed into old age. If he is not struck down in midlife by one of the increasingly common smoking related or cardiovascular diseases, he is likely to survive to a dignified and respected old age, lovingly tended by his wife, sisters, daughters, or daughter in law, secure in the knowlege that, even in the event of total physical or mental disability, he will not be put in an old people's home.

You may well ask where Greek women stand in this male dominated landscape. Alas, they are well down the European female longevity league, in eighth position, surviving only a modest $5 \cdot 2$ years longer than their men, instead of the $\mathbf{8 . 2}$ difference in survival experienced by the French. But surely it is this male-female mortality differential that we should be striving to reduce, together with improving the quality of life gained for both older men and women? JUDITH HOLLIS-TRIANTAFILLOU, general practitioner, Athens 